\title{
Expression of glucocorticoid receptor and glucose transporter-1 during placental development in the diabetic rat
}

\author{
Emin Türkay Korgun ${ }^{1}$, Nuray Acar ${ }^{1}$, Leyla Sati ${ }^{1}$, Dijle Kipmen-Korgun ${ }^{2}$, \\ Asl1 Ozen ${ }^{1}$, Gozde Unek ${ }^{1}$, İsmail Ustunel ${ }^{1}$, Ramazan Demir ${ }^{1}$ \\ ${ }^{1}$ Department of Histology and Embryology, Medical Faculty, Akdeniz University, Antalya, Turkey \\ ${ }^{2}$ Department of Biochemistry, Medical Faculty, Akdeniz University, Antalya, Turkey
}

\begin{abstract}
In various tissues, glucocorticoids (GCs) are known to downregulate glucose transport systems; however, their effects on glucose transporters (GLUTs) in the placenta of a diabetic rat are unknown. Glucocorticoid hormone action within the cell is regulated by the glucocorticoid receptor (GR). Thus, this study was designed to investigate the relationship between GR and glucose transporter expression in the placenta of the diabetic rat. Our immunohistochemical results indicated that GR and glucose transporter protein 1 (GLUT 1) are expressed ubiquitously in the trophoblast and endothelial cells of the labyrinthine zone, where maternal fetal transport takes place in the rat placenta. Expression of GR in the junctional zone of the rat placenta was detected in giant cells, and in some spongiotrophoblast cells, but not in the glycogen cells. GLUT 1 was present, especially in glycogen cells during early pregnancy, and in the spongiotrophoblast cells of the junctional zone during late pregnancy. Amounts of GR and GLUT 1 protein were increased towards the end of gestation both in the control and the diabetic placenta. However, at days 17 and 19 of gestation, only the placental GR protein was significantly increased in the streptozotocin-induced diabetic rats compared to control rats. Diabetes led to a significant decrease in placental weight at gestation day 15. In contrast, at gestational days 17 and 21, the weights of the diabetic placenta were significantly increased as compared with the controls. Moreover, diabetes induced fetus intrauterine growth retardation at gestational days 13,17 and 21 . In conclusion, the localization pattern of GR and GLUT 1 proteins in the same cell types led us to believe that there might be a relationship between GR and GLUT 1 expressions at the cellular level. GLUT 1 does not play a pivotal role in diabetic pregnancies. However, placental growth abnormalities during diabetic pregnancy may be related to the amount of GR. (Folia Histochemica et Cytobiologica 2011; Vol. 49, No. 2, pp. 325-334)
\end{abstract}

Key words: placental development, diabetes, glucocorticoid receptor, glucose transport

\section{Introduction}

The placenta, a complex organ of limited life-span whose metabolic and endocrine activities are not yet fully understood, is the organ responsible for the

Correspondence address: E.T. Korgun,

Department of Histology and Embryology,

Medical Faculty, Akdeniz University,

07070 Antalya, Turkey;

tel./fax: (+ 90 242) 22744 86;

e-mail:korgun@akdeniz.edu.tr transfer of nutrients and waste products between the fetal and maternal circulations. The placenta must integrate signals from the fetus and the mother in an attempt to match fetal demand with maternal substrate supply [1]. Therefore, it plays a pivotal role in fetal growth. The failure of placental growth and development during early and middle stages of pregnancy is directly associated with decreased fetal growth in late pregnancy [2].

Rat placenta is composed of two main regions, the junctional zone (JZ) and the labyrinthine zone (LZ). The LZ is located at the fetal interface and 
comprises stem cells capable either of differentiating into trophoblast giant cells, or of fusing to form syncytial trophoblast cells. Labyrinthine trophoblast giant cells possess a restricted capacity for hormone production [3], whereas syncytial trophoblast cells mediate the transfer of nutrients and wastes between maternal and fetal compartments [4]. The JZ represents the interface between the maternal and fetal tissues, and is the site of trophoblast proliferation. The LZ of the trophoblast consists of three morphologically distinct cell types: trophoblast giant cells, spongiotrophoblast cells, and glycogen cells [5].

Trophoblast giant cells arise by endoreduplication, are situated at the maternal/placental interface, and are one of the major endocrine cells of the placenta [3]. They synthesize and secrete steroid and peptide hormones. Spongiotrophoblast cells are located immediately beneath the trophoblast giant cell layer, and synthesize and secrete peptide hormones. Glycogen cells are a transient cell type embedded among the spongiotrophoblast cells. Beyond their accumulation of glycogen, their biology is not well understood [3].

Glucose, a primary energy substrate for fetal and placental development, is the principal carbohydrate to be transported by the placenta. It is transferred across the placenta by sodium-independent facilitated diffusion along a concentration gradient $[6,7]$. In general, a family of transporter proteins mediates this process. These glucose transporters (GLUTs) are characterized by the presence of 12 membrane-spanning helices and several conserved sequence motifs. Twelve different facilitative glucose or fructose transporter isoforms, GLUT 1-GLUT 12, have been cloned [8]. GLUT proteins show a tissuespecific distribution. The predominant form expressed in the placenta during early pregnancy is GLUT 1, the tissue-barrier isoform seen in trophoblastic cell plasma membranes $[9,10]$.

On the other hand, the placenta is a glucocorticoid target organ per se, with placental growth $[11,12]$ and endocrine function known to be affected by GCs [13]. Glucocorticoid hormone action within the cell is regulated by expression of the GR and isoforms of $11 \beta$-hydroxysteroid dehydrogenase 1 and 2 (11 $\beta$ HSD1 and $11 \beta$ HSD2) [14-16]. Therefore, many classical effects of GCs are mediated by GR [14]. Fetal GCs are derived from the mother and are present in low concentrations until the end of gestation. The transplacental GCs concentration gradient is maintained by placental $11 \beta$ HSD2, which converts the active GCs, cortisol and corticosterone, to their inactive metabolites [17]. Consequently, precise control of the transplacental transfer of maternal glucocorticoids to the fetus is critical for placental and fetal development. GCs could potentially affect a wide range of cellular functions within the embryo and placenta, from trophoblast differentiation soon after implantation, to hormone synthesis in the fully differentiated placenta [18].

Additionally, GCs specifically inhibit glucose transport in a variety of peripheral tissues, such as skeletal muscle, adipocytes, and endothelial cells [19-21]. Furthermore, placental glucose transporters expressions are regulated by GCs [22].

Maternal diabetes is one of the commonest complications during pregnancy. The altered metabolic environment in maternal diabetes during pregnancy interferes with normal placental and fetal development. In humans, as well as in rodents, there is an increased incidence of congenital malformations and fetal growth retardation. The exposure of the placenta to a diabetic environment may lead to functional and structural derangements, including villous immaturity, enlargement of the surface area of exchange and thickening of the basement membrane [1].

In short, inadequate transfer of compounds between mother and fetus can cause abnormalities in the developing fetus. This means that studies during placentation in diabetic rats are of great importance. It is hypothesized that GCs regulate placental glucose transport in many cell types and tissues. Therefore, the aim of this study was to investigate the relationship between glucose transport and the glucocorticoid metabolism in rat placental development of normal and diabetic pregnancy, focusing on the GR and GLUT 1 protein.

\section{Material and methods}

Animals. Female albino rats (Rattus norvegicus) (250-270 g) were kept at a temperature of $22 \pm 2^{\circ} \mathrm{C}$ and maintained on a cycle of 12 hours light/12 hours darkness, with free access to food and water. The rats had been kept in cages, as with two female per one male rat. The onset of pregnancy was determined by vaginal smear inspection to see spermatozoa after overnight mating. The day when a positive smear was found was designated day 0 of pregnancy. Diabetes mellitus was experimentally induced by a single intraperitoneal (i.p.) injection of $60 \mathrm{mg} / \mathrm{kg}$ body weight streptozotocin (STZ, Sigma Chemical Co. S 0130) 50 mM sodium citrate buffer, $\mathrm{pH} 4.5$ on day six of gestation. Control rats received an equal volume of citrate buffer. After two days of STZ injection, diabetes was confirmed when blood glucose concentration was measured to be greater than 250 $\mathrm{mg} / \mathrm{dl}$. On days 13, 15, 17, 19 and 21 of pregnancy, rats were anesthetized with ether. The experimental design and number of animals, placentas and embryos is shown in Table 1. The anterior abdominal wall was opened and placentas and fetuses were collected and rapidly weighed. Some of the 
Table 1. Experimental design and number of animals, placentas and embryos

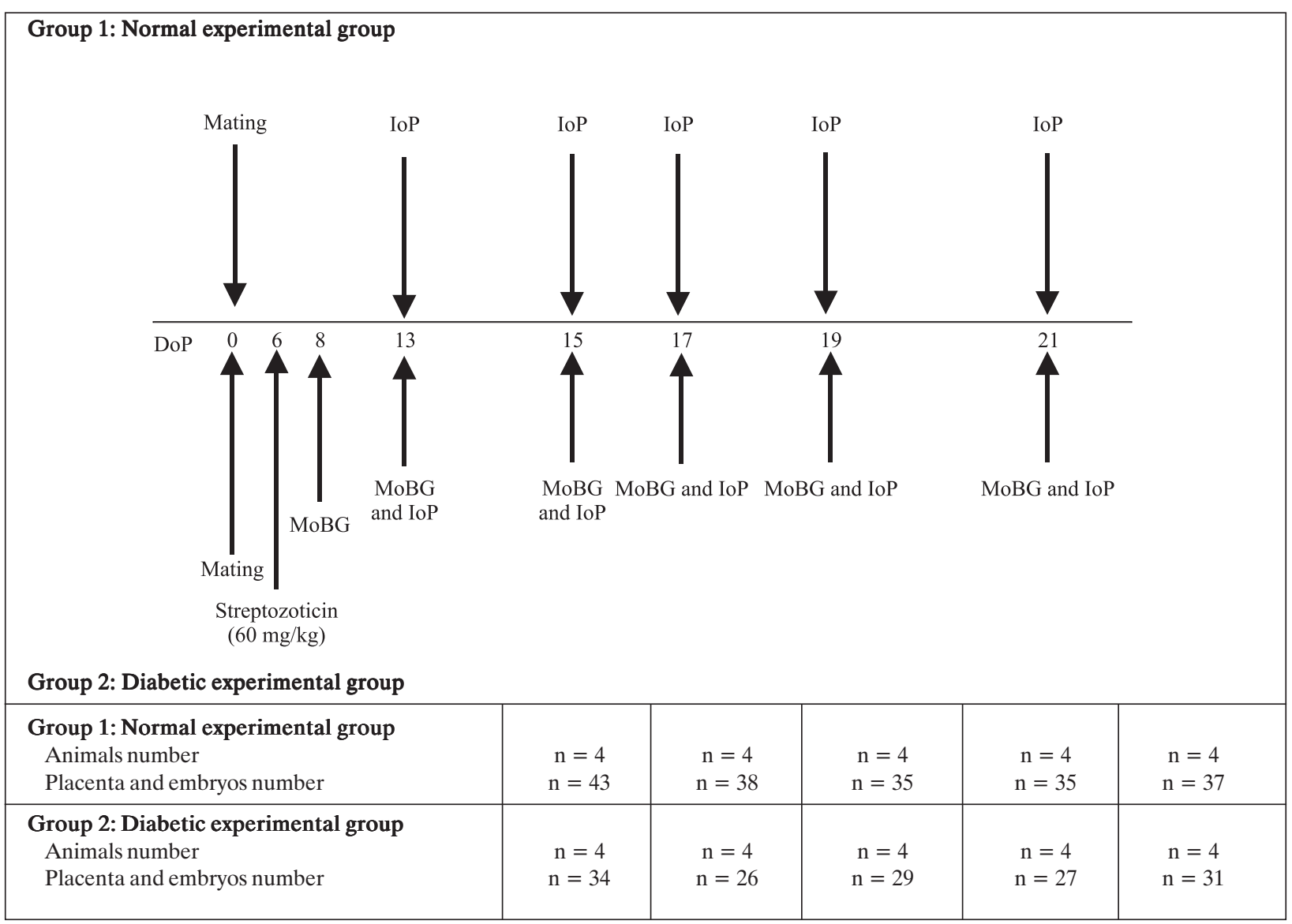

MoBG — measurement of blood glucose; IoP — interruption of pregnancy; DoP — days of pregnancy

placentas were excised and frozen in liquid nitrogen, and kept at $-80^{\circ} \mathrm{C}$ until required for Western blots. The remaining placentas were fixed for four hours in Holland's fixative for immunohistochemistry.

Immunohistochemistry. Immunohistochemistry was performed on paraffin-embedded tissue sections of the developing diabetic and control rat placentas between gestational days (GD) 13 and 21.

The paraffin-embedded serial sections $(6 \mathrm{~mm})$ were collected on SuperFrost Plus slides (Novoglas, Berne, Switzerland). After re-hydration, samples were transferred to $0.01 \mathrm{M}$ citrate buffer $(\mathrm{pH}=6)$ and subsequently heated in a microwave oven for $2 \times 5$ minutes at $750 \mathrm{~W}$ for antigen-retrieving. After cooling for 20 minutes at room temperature, the sections were washed with PBS. To remove endogenous peroxidase activity, sections were kept in $3 \% \mathrm{H}_{2} \mathrm{O}_{2}$ for 20 minutes, and afterwards washed with PBS. Sections were incubated for 60 minutes at room temperature in a moist chamber with two rabbit antisera one against to C-terminal sequences of GLUT 1 (AB 1340 from Chemicon, Temecula, CA, USA) and the other two N-terminal sequences of GR (Sc-1004 from Santa Cruz Biotechnology, Santa Cruz, CA, USA). Antisera were diluted 1:1,000 (GR), and 1:2,000 (GLUT 1) with Antibody Diluent (Dako, Carpinteria, CA, USA). Labeling was visualized using the Universal LSAB 2 Kits (Dako, $\mathrm{K}$ 0609) according to the instructions of the manufacturer. The sections were counterstained with Mayers's hemalum (Merck, Darmstadt, Germany) and mounted with Kaiser's glycerol gelatin (Merck). For controls, sections were incubated with rabbit serum (Dako X 0902). Photographs were taken with an Axiophot microscope (Zeiss, Oberkochen, Germany).

Investigations were conducted in accordance with the Guide for the Care and Use of Laboratory Animals (Institute for Laboratory Animal Research of the National Academy of Science, Bethesda, MD, USA, 1996).

\section{SDS-polyacrylamide gel electrophoresis and Western} blotting. Cellular proteins from diabetic and control rat placenta were solubilized in Laemmli sample buffer (Sigma) supplemented with Complete ${ }^{R}$ protease inhibitor cocktail (Boehringer, Mannheim, Germany). Insoluble material was removed by centrifugation at $100,000 \times \mathrm{g}$ for 1 hour at $4^{\circ} \mathrm{C}$. Samples were stored at $-70^{\circ} \mathrm{C}$. Prior to electrophoresis, samples were boiled for three minutes at $100^{\circ} \mathrm{C}$. 
Amounts of protein were determined according to Lowry et al. [23] and $50 \mu \mathrm{g}$ protein was applied to each lane. Samples were subjected to SDS-polyacrylamide gel electrophoresis on 8-18\% gradient gels (ExcelGel; Pharmacia, Uppsala, Sweden) using SDS buffer strips (ExcelGel, Pharmacia). Samples were run for 150 minutes at a constant $600 \mathrm{~V} / 50 \mathrm{~mA} / 30 \mathrm{~W}$. Proteins were transferred onto nitrocellulose membranes (Pharmacia) by semi-dry electroblotting in a buffer containing $0.2 \mathrm{~mol} / \mathrm{l}$ glycine, $25 \mathrm{mM}$ Tris and $20 \%$ methanol for 45 minutes at $30 \mathrm{~V} / 100 \mathrm{~mA} / 6 \mathrm{~W}$. Successful transfer was confirmed by Ponceau S (Sigma) staining of the blots. The membranes were blocked for 1 hour with 5\% non-fat dry milk (Bio-Rad, Hercules, CA, USA) and 0.1\% Tween-20 (Sigma) in $0.14 \mathrm{~mol} / 1$ Tris-buffered saline (TBS) $\mathrm{pH} 7.2-7.4$ at $4^{\circ} \mathrm{C}$. The same solution was used for subsequent washing steps and as a diluent for the antibodies. The blotting membranes were incubated overnight at $4^{\circ} \mathrm{C}$ with rabbit antisera against GLUT $1(1: 3,000)$ and GR (1:200). After washing, the membranes were further incubated with goat anti-rabbit IgG horseradish peroxidase conjugate (Bio-Rad) diluted 1:2,000 for 1 hour at room temperature. After three washing steps in TBS, pH 7.2-7.4, immunolabeling was visualized using the chemiluminescencebased SuperSignal CL-HRP Substrate System (Pierce, Rockford, IL, USA) according to the manufacturer's instructions. Membranes were exposed to Hyperfilm (Amersham), which was subsequently scanned using an Alpha Digi Doc ${ }^{\mathrm{TM}}$ densitometer and quantified with AlphaEaseFC software (Alpha Digi Doc 1000). Control blots were incubated with antibody diluent alone.

Statistical analysis. Results are presented as mean \pm standard error of mean (SEM). Comparisons between the controls and the diabetic groups were performed by Mann-Whitney U tests for individual comparisons or Students $t$-test as appropriate. Statistical calculations were performed using Sigmastat Program for Windows, version 2.0 (Jandel Scientific Corporation, San Rafael, CA, USA).

\section{Results}

\section{Measurement of maternal glucose}

Blood glucose level was routinely determined using a glucometer according to the user's guide, with certain modifications. Blood samples were collected from rat tail veins and a drop of blood was applied to the pad of the glucose strips. After $20 \mathrm{sec}-$ onds, the pad surface was carefully blotted dry and inserted into the test slot of the glucometer. We measured the maternal glucose concentration during gestational days $13,15,17,19,21$. The data is shown in Figure 1A.

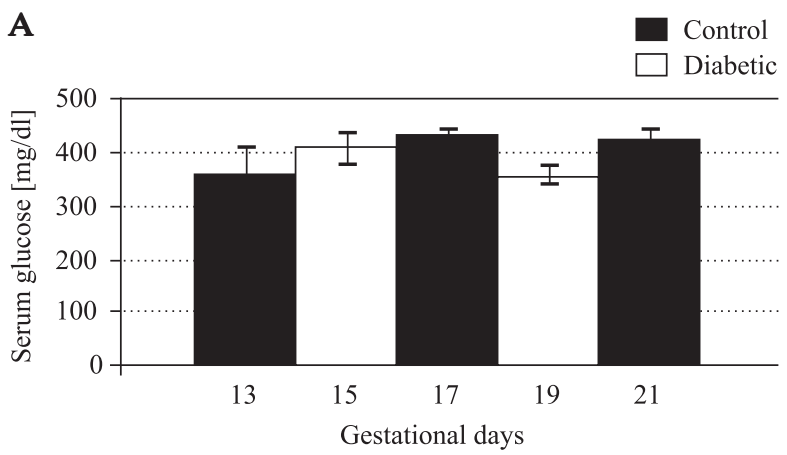

B
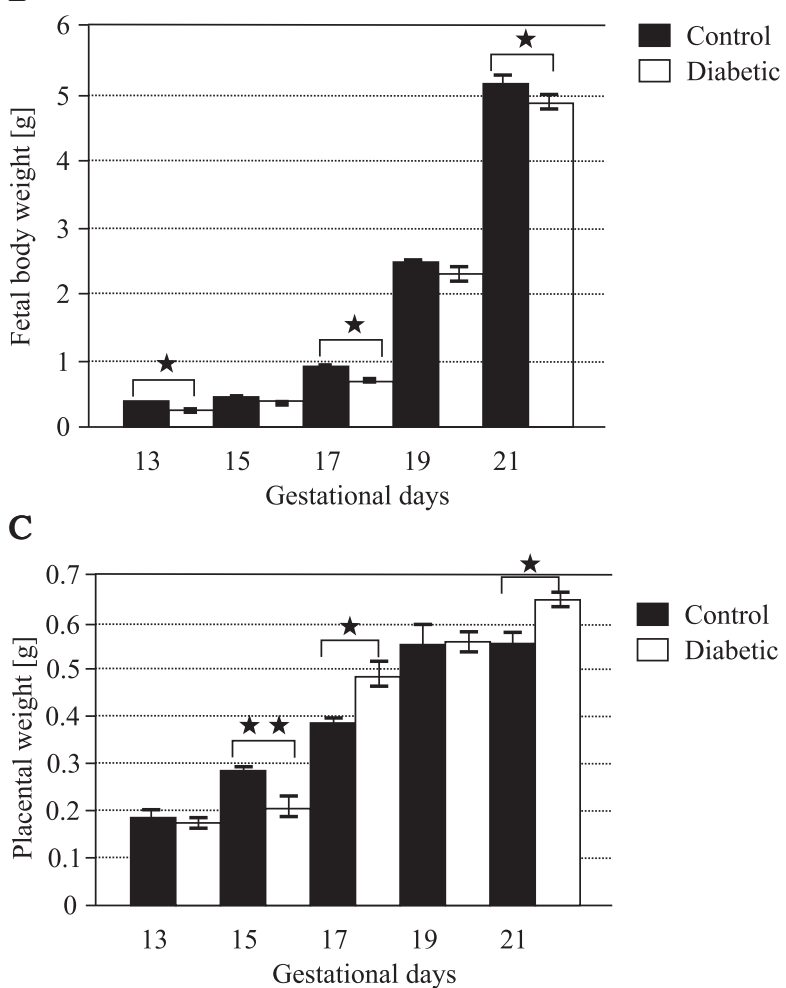

Figure 1. A. Measurements of maternal glucose concentration in STZ-induced diabetic group; B. Fetal body; C. Placental weights of control and STZ-diabetic group from days 13 to 21 of pregnancy. Results are presented as means \pm SEM. Both were tested by the Mann-Whitney U test. Statistically-significant effects of GR expression in the diabetic rat placenta are indicated by: $* * \mathrm{p}<0.005 ; * \mathrm{p}<0.001$

\section{Hyperglycemia effects on placenta and body weights of fetus}

The fetuses and the placentas were weighed from GD13 to GD21. The fetuses of the diabetic rat model showed intrauterine growth retardation compared to the fetuses of the control group. Significant differences were observed in fetal weight between the control and diabetic groups on GD13, GD17 and GD21 ( $\mathrm{p}<0.001$ by Mann-Whitney U test) (Figure $1 \mathrm{~B})$. Placental weights of diabetic mothers were 
significantly lower on GD15 ( $\mathrm{p}<0.05$ by Mann-Whitney U test) and higher on GD17 and GD21 ( $\mathrm{p}<0.001$ by Mann-Whitney U test) than the placental weights from control rats (Figure 1C).

\section{Localization of GR and GLUT 1 proteins by immunohistochemistry}

During the development of the normal and diabetic rat placenta, we determined the immunocytochemical localization of GR and GLUT 1 proteins. The data from the immunohistochemistry is summarized in Table 2. The two observers were always concordant, except for the assessments of weak to moderate staining.

\section{Chorioallantoic placenta}

The chorioallantoic placenta consists of two distinct zones: the LZ, the major site of maternal fetal exchange, and the JZ, the primary site of placental steroid and peptide hormone synthesis.

\section{Localization of $G R$}

On the $13^{\text {th }}$ day of pregnancy of the normal and diabetic rat placenta, there was GR expression mainly in the LZ, but also in the JZ at particularly high levels in the trophoblast cells and endothelial cells surrounding the maternal blood vessels (Figure 2A). From the $15^{\text {th }}$ to the $21^{\text {st }}$ day of pregnancy, GR was detected in decidual cells, giant cells, and some spongiotrophoblast cells of the JZ. Endothelial cells, labyrinth trophoblast cells and labyrinthic giant cells were immunopositive for GR. However, glycogen cells did not react with GR (Figures 2C, E). The expression level of GR was increased, especially in endothelial cells of the LZ and spongiotrophoblast cells of JZ in the diabetic rat placenta (Figures 3C, D) compared to normal placenta (Figures 3A, B) on GD17 and GD19.

\section{Localization of GLUT 1}

GLUT 1 expression was restricted to the LZ, especially in the endothelial cells of fetal vessels on the $13^{\text {th }}$ day of pregnancy (Figure $2 \mathrm{~B}$ ). From the $15^{\text {th }}$ to the $21^{\text {st }}$ day of pregnancy, GLUT 1 was present especially in glycogen cells and some spongiotrophoblast cells from the JZ. Endothelial cells and some trophoblast cells of the LZ were immunopositive for GLUT 1 (Figures 2D, F). The reaction pattern of GLUT 1 remained constant from GD15 to GD 21, with only slight changes in intensity (Table 2).

Expression levels of GLUT 1 were increased towards the end of gestation, especially spongiotrophoblast cells of $\mathrm{JZ}$ and endothelial cells of the $\mathrm{LZ}$ in the diabetic rat placenta (Figures 3E, F). One of the characteristic properties of diabetic placenta is numerous cysts and glycogen cells which are immunopositive for GLUT 1 (Figure 3H) but not GR (Figure 3G).

\section{Yolk sac placenta}

The yolk sac placenta is composed of parietal yolk sac and visceral yolk sac, the second site for maternal-fetal exchange. GR (Figure $2 \mathrm{G}$ ) but not GLUT 1 (Figure 2H) were detected in the fetal mesenchymal cells. Yolk sac epithelium was immunopositive for GLUT 1 (Figure 2H) but not GR (Figure 2G). GLUT 1 was not found in the parietal and visceral endodermal cells, but visceral endodermal cells were immunopositive for GR (data not shown).

\section{Expression of GLUT 1 and GR in the placenta on immunoblots}

GR and GLUT 1 protein expressions as quantified by Western blotting in normal and diabetic rat placentas from GD13 to GD21 are shown in Figures 4A, B. GR and GLUT 1 proteins were detected by Western blot analysis as single bands at the expected sizes of 95 and $54 \mathrm{kDa}$, respectively. Amounts of the control and diabetic placental GR and GLUT 1 proteins increased towards gestational days (Figures 4A, B). However, only the placental GR protein at GD17 and GD19 day was significantly increased in the STZ-diabetic rats compared to the control rats $(\mathrm{p}<0.001$, Figure 4A).

\section{Discussion}

Our study demonstrates, for the first time, the spatial and temporal localization patterns of GR and GLUT 1 proteins in diabetic rat placenta.

Glucocorticoid dynamics during diabetic pregnancy is a controversial issue. Plasma corticosterone levels at term are dependent on the severity of maternal diabetes [24]. Maternal and fetal plasma corticosterone levels of diabetic rats were significantly lower than the corresponding control values between GD19 and GD22 [25]. We detected GR expression to be significantly increased between the $17^{\text {th }}$ and $19^{\text {th }}$ days of pregnancy in diabetic rats. Van Beek et al. [26] established that GCs increase $11 \beta$-HSD2 activity and mRNA expression, but conversely decrease GR mRNA expression in cultured human trophoblast cells. Additionally, treatment of trophoblasts with the GR antagonist RU-486 blocked the glucocorticoid-induced increase of $11 \beta$-HSD2 activity, indicating that the effect of GCs on $11 \beta$-HSD2 is probably mediated by GR [26]. 
Table 2. Distribution of GR and GLUT 1 immunoreactivity in the normal and diabetic rat placenta and uterus at GD13, GD15, GD17, GD19 and GD21 ${ }^{\mathrm{a}}$

\begin{tabular}{|c|c|c|c|c|c|c|c|c|c|c|c|}
\hline 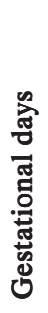 & 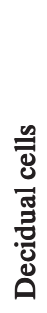 & 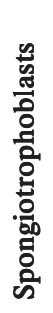 & 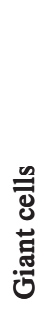 & 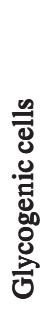 & 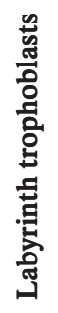 & 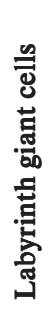 & 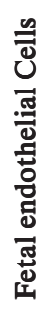 & 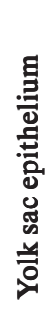 & 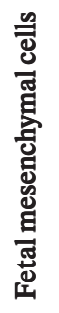 & 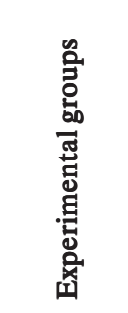 & 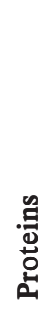 \\
\hline \multirow[t]{2}{*}{13} & 1 & 1 & 2 & 0 & 1 & - & 2 & - & - & Diabetic & \multirow{10}{*}{ 苞 } \\
\hline & 1 & 1 & 2 & 0 & 1 & - & 2 & - & - & Control & \\
\hline \multirow[t]{2}{*}{15} & 1 & 2 & 2 & 0 & 1 & 3 & 2 & 0 & 2 & Diabetic & \\
\hline & 1 & 2 & 2 & 0 & 1 & 2 & 2 & 0 & 2 & Control & \\
\hline \multirow[t]{2}{*}{17} & 1 & 3 & 3 & 0 & 1 & 3 & 3 & 0 & 3 & Diabetic & \\
\hline & 1 & 2 & 2 & 0 & 1 & 2 & 2 & 0 & 2 & Control & \\
\hline \multirow[t]{2}{*}{19} & 1 & 3 & 3 & 0 & 1 & 3 & 3 & 0 & 3 & Diabetic & \\
\hline & 1 & 2 & 2 & 0 & 1 & 2 & 2 & 0 & 2 & Control & \\
\hline \multirow[t]{2}{*}{21} & 1 & 3 & 3 & 0 & 1 & 2 & 3 & 0 & 2 & Diabetic & \\
\hline & 1 & 2 & 2 & 0 & 1 & 2 & 2 & 0 & 2 & Control & \\
\hline \multirow[t]{2}{*}{13} & 1 & 0 & 0 & 0 & 1 & - & 2 & - & - & Diabetic & \multirow{10}{*}{ 卢 } \\
\hline & 1 & 0 & 0 & 0 & 1 & - & 2 & - & - & Control & \\
\hline \multirow[t]{2}{*}{15} & 1 & 1 & 2 & 0 & 1 & 0 & 2 & 1 & 0 & Diabetic & \\
\hline & 1 & 1 & 2 & 0 & 1 & 0 & 2 & 1 & 0 & Control & \\
\hline \multirow[t]{2}{*}{17} & 1 & 1 & 2 & 0 & 2 & 0 & 3 & 1 & 0 & Diabetic & \\
\hline & 1 & 1 & 2 & 0 & 2 & 0 & 3 & 1 & 0 & Control & \\
\hline \multirow[t]{2}{*}{19} & 1 & 3 & 2 & 0 & 2 & 0 & 3 & 1 & 0 & Diabetic & \\
\hline & 1 & 2 & 1 & 0 & 2 & 0 & 3 & 1 & 0 & Control & \\
\hline \multirow[t]{2}{*}{21} & 1 & 3 & 2 & 0 & 2 & 0 & 3 & 1 & 0 & Diabetic & \\
\hline & 1 & 2 & 1 & 0 & 2 & 0 & 3 & 1 & 0 & Control & \\
\hline
\end{tabular}

a This table was designed according to the percentage of positively stained cell count; $3,2,1$, and 0 indicate the differences in signal intensities observed by optical microscopy and reflect levels of GR and GLUT 1 proteins: $0=$ absent; $1=$ weak expression $(10-30 \%) ; 2=$ moderate expression (40-60\%); and $3=$ high expression (70-100\%). Empty cells in the table indicate the concerned tissue is either not yet differentiated or has not been investigated at the respective gestational days

In this study, we failed to detect $11 \beta$-HSD2 in rat placenta during pregnancy, but a previous study showed $11 \beta$-HSD2 gene expression to be decreased in placentas of STZ-induced diabetic pregnant rats [27]. Our data demonstrating the increased levels of GR protein in the placentas of STZ-induced diabetic rats in late pregnancy may indicate that $11 \beta$-HSD 2 is mediated by GR, similar to the results obtained by Van Beek et al. [26]. Our data suggests that diabetic rat placentas show increased expression towards the GR and sustain the catabolism of corticosterone.

We found that GR protein expression in endothelial cells of the LZ was associated with the maternal blood vessels; thus GR is ideally located for its proposed role in regulating the transplacental passage of maternal glucocorticoid to the fetus by the mechanism to protect the fetal compartment from high levels of maternal glucocorticoids. Expression of GR protein was detected in both labyrinthic and junctional layers of rat placenta, indicating these two important sites are glucocorticoid target sites. Therefore, the co-expression of GLUT 1 with GR protein in trophoblast cells of the LZ and in the spongiotrophoblast cells of the JZ may suggest that GR activity might participate in regulating local glucocorticoid-mediated cellular differentiation, placental growth and function, such as expressions of glucose transporters proteins.

GR was abundantly expressed in the spongiotrophoblast and giant cells of the JZ. Therefore, GCs may regulate placental synthesis of both steroid and 

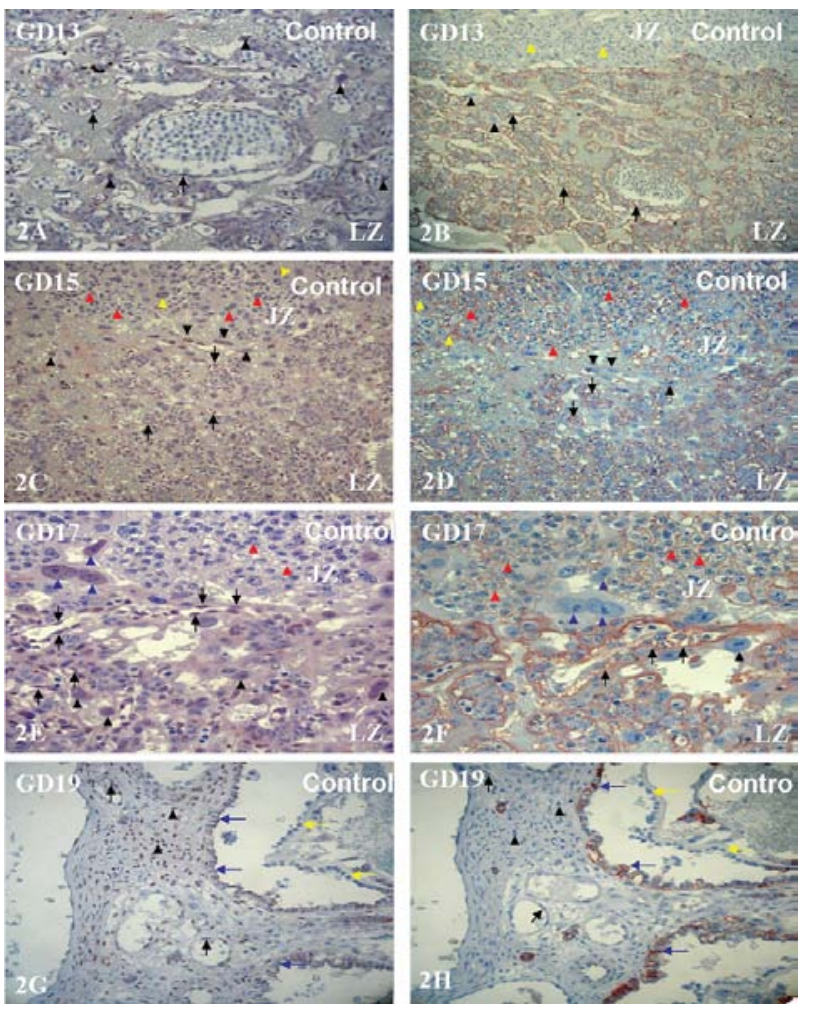

Figure 2. Transverse sections of the developing rat placenta from GD13 (A, B), GD15 (C, D), GD17 (E, F), and GD19 $(\mathbf{G}, \mathbf{H})$. A. GR. Arrowheads point to labyrinthine trophoblast, arrows point to fetal endothelial cells in the LZ. B. GLUT 1. Arrows point to fetal endothelial cells. Arrowheads indicate an immunonegative labyrinthine trophoblast and yellow arrowheads indicate immunonegative spongiotrophoblast cells. Inset shows the negative control staining for GLUT 1. C. GR. Arrowheads point to labyrinthine trophoblast, arrows point to fetal endothelial cells, yellow arrowheads indicate spongiotrophoblast cells and red arrowheads indicate an immunonegative glycogen cell. D. GLUT 1. Red arrowheads point to glycogen cells, arrows point to fetal endothelial cells, yellow arrowheads indicate spongiotrophoblast cells and arrowheads indicate labyrinthine trophoblast. E. GR. Arrowheads point to labyrinthine trophoblast, blue arrowheads point to giant cells, arrows point to fetal endothelial cells, and red arrowheads indicate immunonegative glycogen cells. F. GLUT 1. Red arrowheads point to glycogen cells, arrows point to fetal endothelial cells, arrowheads indicate labyrinthine trophoblast cells, yellow arrowheads indicate spongiotrophoblast cells and blue arrowheads indicate immunonegative giant cells. G. GR. arrowheads point to fetal mesenchymal cells, arrows point to endothelial cells of the umbilical vessels, blue arrows point to immunonegative yolk sac epithelial cells, and yellow arrows indicate immunonegative parietal cells on the reichert membrane. H. GLUT 1. Arrowheads point to immunonegative fetal mesenchymal cells, blue arrows point to yolk sac epithelial cells, arrows point to immunonegative endothelial cells of the umbilical vessels, and yellow arrows indicate immunonegative parietal cells on the reichert membrane

GD - gestational day; JZ — junctional zone; LZ — labyrinth zone. Magnification $\times 300$ for B, C, D, G, H; $\times 600$ for A, E, F
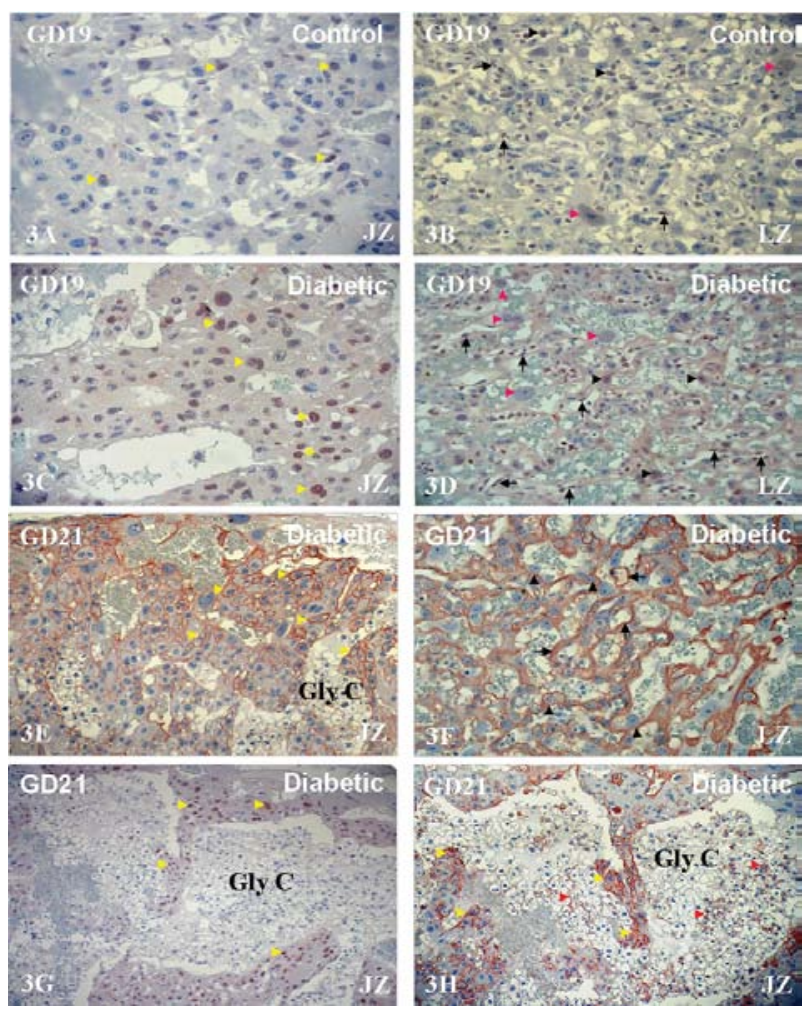

Figure 3. Transverse sections of the control (A, B) and diabetic rat placenta from GD19 (C, D) and GD21 (E, F). A. GR. Yellow arrowheads indicate spongiotrophoblast cells. B. GR. Arrowheads point to labyrinth trophoblast, blue arrowheads point to labyrinth giant cells and arrows point to fetal endothelial cells. C. GR. Yellow arrowheads indicate spongiotrophoblast cells. D. GR. Arrowheads point to labyrinthine trophoblast, blue arrowheads point to labyrinthine giant cells and arrows point to fetal endothelial cells. E. GLUT 1. Yellow arrowheads indicate spongiotrophoblast cells. F. GLUT 1. Arrowheads point to basement membrane of labyrinthine trophoblast cells; arrows indicate basement membrane of fetal endothelial cells. G. GR. Yellow arrowheads indicate spongiotrophoblast cells. Inset shows the negative control staining for GR. H. GLUT 1. Yellow arrowheads indicate spongiotrophoblast cells and red arrowheads point to glycogen cells

GD — gestational day; JZ — junctional zone; LZ — labyrinth zone; Gly C - glycogen cell. Magnification $\times 300$ for $\mathbf{G}, \mathbf{H} ; \times 600$ for A-F

peptide hormones. During diabetes, several metabolic adjustments take place to tailor high circulating blood glucose levels. Glucocorticoid and GR might play a critical role in such metabolic conditions.

In various tissues, GCs down-regulate glucose transport systems; however, their action on glucose transport systems in placenta is unknown, especially in the diabetic placenta. Previous studies found that, in rats treated with GCs from day 15 to day 21 of pregnancy, placental GLUT 1 and GLUT 3 protein expressions increased dose-dependently. Increased 
A

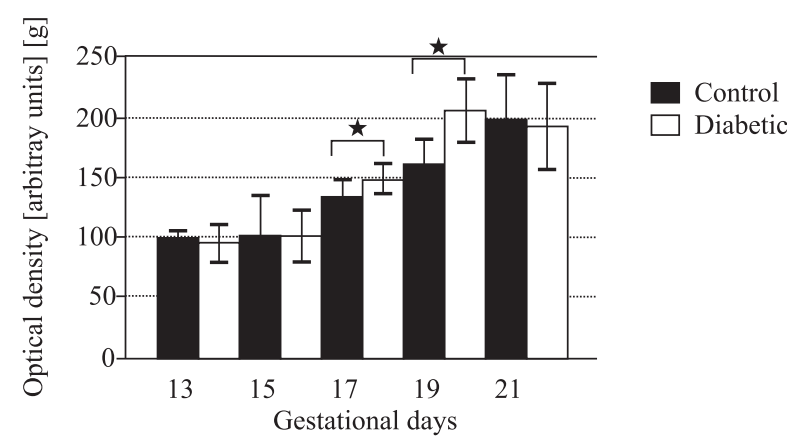

13N 13D 15N 15D 17N 17D 19N 19D21N 21D GR protein $95 \mathrm{kDa}-\ldots+\ldots$

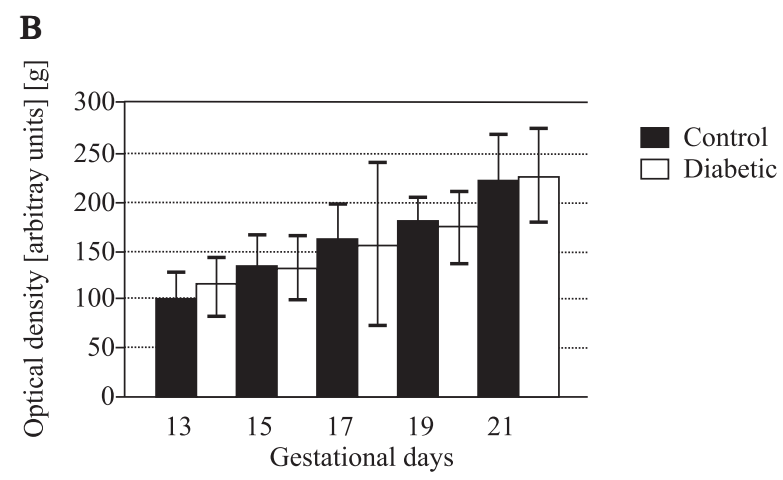

13N 13D 15N15D 17N 17D19N 19D21N 21D

GLUT1

protein $<55 \mathrm{kDa}$

Figure 4. A. Western blot analysis of GR from control and diabetic placenta. Values are expressed as mean \pm \pm SEM of ratio of control GD13 GR levels. Statistically significant effects of GR expression in the diabetic rat placenta are indicated by: $* \mathrm{p}<0.001$. B. Western blot analysis of GLUT 1 from control and diabetic placenta. Values are expressed as mean \pm SEM of ratio of control GD13 GLUT 1 levels. Statistically significant effects of GLUT 1 expression in the diabetic rat placenta are not indicated

placental GLUT 1 and GLUT 3 protein expressions may reflect an attempt to increase placental or fetal glucose supply to attenuate the effect of excessive exposure to GCs in diminishing fetal growth [28]. In contrast, both GLUT 1 and GLUT 3 transcripts and proteins were significantly downregulated in isolated human trophoblast cells and in rat placentas by exposure to GCs [22]. The results of previous studies show that expressions of GLUT 1 and GLUT 3 proteins in the rat placenta are differentially affected by $\mathrm{GC}$ treatment. Our result regarding the protein levels of GR and GLUT 1 showed that the glucose transport system was working independently from GR protein expression in normal and diabetic placenta. How- ever, both GR and GLUT 1 proteins were expressed in spongiotrophoblast cells, endothelial cells and trophoblast cells at the same time. So, there may also be an autocrine regulation between GR and GLUT 1.

Ogura et al. [29] demonstrated that GLUT 1 protein in placentas from diabetic mice was significantly decreased compared to controls. In spite of this, we determined that the expression level of GLUT 1 protein was not different in the developing normal rat placenta compared to diabetic placenta. Other reports have found no significant change in rat placental GLUT 1 protein levels after STZ [30, 31] and glucose [32] treatment, mirroring our data. Conversely, GLUT 3 mRNA and protein expressions in diabetic rat placentas increased compared to controls [30,31]. Our immunohistochemical results showed that GLUT 1 protein was localized mainly in the membranes on both sides of the trophoblast cells that predominantly line the labyrinthine maternal vascular spaces, and abundantly in the fetal endothelial cells. These findings suggest that GLUT 1 expression in the rat placenta is specifically localized in cells responsible for transplacental glucose transport. On the other hand, we showed for the first time that there is a higher expression of GLUT 1 in the glycogen cells in early pregnancy compared to late pregnancy. This phenomenon may protect fetuses from glucose toxicity and deposit glucose as glycogen, an energy resource for placental development.

The placental weights of STZ induced diabetic rats at term are higher than those of control animals [33]. By contrast, the weight and nucleic acid content of diabetic rat placenta on days 14 and 16 of gestation are significantly lower [34]. We found placental weights of diabetic mothers were significantly lower on GD15 and higher on GD17 and GD21 ( $\mathrm{p}<0.001$ by Mann-Whitney U test) than placental weights from control rats (Figure 1C). Our data supported the previous studies and suggests that perhaps glucose inhibits proliferation in the diabetic placental cells in the early gestational stage, and as a result placental growth diminished. The proliferative activity of diabetic placental cells was found to be high on GD17 and GD21.

The fetuses of our diabetic model showed intrauterine growth retardation compared to fetuses of the control group. These observations showed that placental GLUT 1 protein expression could not be stimulated under hyperglycemic conditions. Thus, perhaps another glucose transporter isoform, such as GLUT 3, may play a pivotal role in the severe alterations of placental function observed in diabetic pregnancies.

Our findings indicated that STZ-induced diabetes increased the activation of placental GR in late 
pregnancy that might influence the differentiation process via GCs in placenta. The precise mechanisms of GR regulation in STZ-induced diabetes are, however, unclear. Moreover, it will be of much clinical significance, in future, to unravel the correlation between STZ-induced diabetes and the regulation of GR function. Further investigation is necessary to clarify the role of GR in the diabetic rat placental glucose transport mechanism.

In conclusion, the localization pattern of GR and GLUT 1 proteins in the same cell types led us to believe that there might be a relationship between GR and GLUT 1 expressions at the cellular level. GLUT 1 does not play a pivotal role in diabetic pregnancies. However, placental growth abnormalities during diabetic pregnancy may be related to the amount of GR.

\section{Acknowledgements}

The authors are grateful to the Akdeniz University Research Fund for financial support (2003.01.0103.009) and the help of Lynne Vigue, MS, in the preparation of this manuscript.

\section{References}

1. Desoye G, Shafrir E. The human placenta in diabetic pregnancy. Diabetes Rev. 1996;4:70-89.

2. Hay WW, Catz CS, Grave GD, Yaffe SJ. Workshop summary: fetal growth: its regulation and disorders. Pediatrics. 1997;99:585-591.

3. Soares MJ, Chapman BM, Rasmussen CA, Dai G, Kamei T, Orwig KE. Differentiation of trophoblast endocrine cells. Placenta. 1996;17:277-289.

4. Knipp GT, Audus KL, Soares MJ. Nutrient transport across the placenta. Adv Drug Deliv Review. 1999;38:41-58.

5. Davies J, Glasser SR, Histological and fine structural observations on the placenta of the rat. Acta Anat (Basel). 1968; 69:542-608.

6. Bissonnette JM, Hohimer AR, Cronan JZ, Black JA. Glucose transfer across the intact guinea-pig placenta. J Dev Physiol. 1979;1:415-426.

7. Johnson LW, Smith CH. Monosaccharide transport across microvillous membrane of human placenta. Am J Physiol. 1980;238:C160-168.

8. Joost HG, Bell GI, Best JD et al. Nomenclature of the GLUT/ /SLC2A family of sugar/polyol transport facilitators. Am J Physiol Endocrinol Metab. 2002;282:E974-976.

9. Devaskar SU, Devaskar UP, Schroeder RE, Demello D, Fiedorek FT Jr, Mueckler M. Expression of genes involved in placental glucose uptake and transport in the non obese diabetic mouse pregnancy. Am J Obs Gyn. 1994;171:1316-1323.

10. Takata K. Structural basis of glucose transport in the placental barrier: role of GLUT 1 and the gap junction. Endocrine $J$. 1994;41:S3-S8.

11. Reinisch JM, Simon NG. Karow WG, Gandelman R. Prenatal exposure to prednisone in humans and animals retards intrauterine growth. Science. 1978;4366:436-438.

12. Burton PJ, Waddell BJ. 11 beta-Hydroxysteroid dehydrogenase in the rat placenta: developmental changes and the ef- fects of altered glucocorticoid exposure. $J$ Endocrinol. 1994;143:505-513.

13. Karalis K, Goodwin G, Majzoub JA. Cortisol blockade of progesterone: a possible molecular mechanism involved in the initiation of human labor. Nat Med. 1996;2:556-560.

14. Bamberger CM, Schulte HM, Chrousos GP. Molecular determinants of glucocorticoid receptor function and tissue sensitivity to glucocorticoids. Endocr Rev. 1996;17:245-261.

15. Stewart PM, Krozowski ZS. 11 beta-Hydroxysteroid dehydrogenase. Vitam Horm. 1999;57:249-324.

16. Mark PJ, Augustus S, Lewis JL, Hewitt DP, Waddell BJ. Changes in the placental glucocorticoid barrier during rat pregnancy: impact on placental corticosterone levels and regulation by progesterone. Biol Reprod. 2009;80: 1209-1215.

17. Seckl JR. Glucocorticoid programming of the fetus; adult phenotypes and molecular mechanisms. Mol Cell Endocrinol. 2001;185:61-71.

18. Korgun ET, Dohr G, Desoye G, Demir R, Kayisli UA, Hahn T. Expression of insulin, insulin-like growth factor I and glucocorticoid receptor in rat uterus and embryo during decidualization, implantation and organogenesis. Reproduction. 2003;125:75-84.

19. Weinstein SP, Wilson CM, Pritsker A, Cushman SW. Dexamethasone inhibits insulin-stimulated recruitment of GLUT4 to the cell surface in rat skeletal muscle. Metabolism. 1998;47:3-6.

20. Hajduch E, Hainault I, Meunier C et al. Regulation of glucose transporters in cultured rat adipocytes: synergistic effect of insulin and dexamethasone on GLUT4 gene expression through promoter activation. Endocrinology. 1995; 136:4782-4789.

21. Olgemoller B, Schon J, Wieland $\mathrm{OH}$. Endothelial plasma membrane is a glucocorticoid-regulated barrier for the uptake of glucose into the cell. Mol Cell Endocrinol. 1985; 43:165-171.

22. Hahn T, Barth S, Graf R et al. Placental glucose transporter expression is regulated by glucocorticoids. J Clin Endocrinol Metab. 1999;84:1445-1452.

23. Lowry OH, Rosebrough NJ, Farr AL, Randall R. Protein measurement with the Folin phenol reagent. J Biol Chem. 1951;193:265-275.

24. Conliffe P, Mulay S. Effects of insulin, glucose and ACTH on corticosterone production by fetal adrenal cells from diabetic rats. J Endocrinol. 1989;120:393-399.

25. Mulay S, Solomon S. Influence of streptozotocin-induced diabetes in pregnant rats on plasma corticosterone and progesterone levels and on cytoplasmic glucocorticoid receptors in fetal tissues. J Endocrinol. 1983;96:335-345.

26. Van Beek JP, Guan H, Julan L, Yang K. Glucocorticoids stimulate the expression of 11beta-hydroxysteroid dehydrogenase type 2 in cultured human placental trophoblast cells. J Clin Endocrinol Metab. 2004;89:5614-5621.

27. Fujisawa Y, Nakagawa Y, Ren-Shan L, Ohzeki T. Streptozotocin-induced diabetes in the pregnant rat reduces 11 beta-hydroxysteroid dehydrogenase type 2 expression in placenta and fetal kidney. Life Sci. 2004;75:2797-2805.

28. Langdown ML, Sugden MC. Enhanced placental GLUT 1 and GLUT3 expression in dexamethasone-induced fetal growth retardation. Mol Cell Endocrinol. 2001;185: 109-117.

29. Ogura K, Sakata M, Yamaguchi M, Kurachi H, Murata Y. High concentration of glucose decreases glucose transporter-1 expression in mouse placenta in vitro and in vivo. $J$ Endocrinol. 1999;160:443-452. 
30. Boileau P, Mrejen C, Girard J, Hauguelde MS. Overexpression of GLUT3 placental glucose transporter in diabetic rats. J Clin Invest. 1995;96:309-317.

31. Das UG, Sadiq HF, Soares MJ, Hay WW Jr, Devaskar SU. Time-dependent physiological regulation of rodent and ovine placental glucose transporter (GLUT-1) protein. Am J Physiol. 1998;274:R339-347.

32. Ericsson A, Säljö K, Sjöstrand E et al. Brief hyperglycaemia in the early pregnant rat increases fetal weight at term by stimulating placental growth and affecting placental nutrient transport. J Physiol. 2007;581: 1323$-1332$.

33. Aerts L, Holemans K, Van Assche FA. Maternal diabetes during pregnancy: consequences for the offspring. Diabetes Metab Rev. 1990;6:147-167.

34. Robinson J, Canavan JP, Elhaj AJ, Goldspink DF. Maternal diabetes in rats . I. Effect on placental growth and protein turnover. Diabetes. 1988;37:1665-1670

Submitted: 20 November, 2010 Accepted after reviews: 30 January, 2011 